\title{
A Levy-type solution for buckling analysis of micro-plates considering the small length scale
}

\author{
H.R. NOORI ${ }^{1}$ AND E. JOMEHZADEH ${ }^{2, a}$ \\ 1 Department of Mechanical Engineering, Islamic Azad University, Jiroft Branch, Jiroft, Kerman, Iran \\ 2 Department of Mechanical Engineering, Graduate University of Advanced Technology, Kerman, Iran
}

Received 18 November 2013, Accepted 14 April 2014

\begin{abstract}
In this paper, a Levy-type solution based on the modified couple stress theory is developed to study the buckling behaviors of micro-plates. Based on this theory, length scale parameter is considered to capture the size effect of rectangular micro-plates. Minimum potential energy and adjacent-equilibrium criteria are exploited to obtain the stability equations and corresponding boundary conditions. Different boundary conditions with two opposite edges simply supported and arbitrary boundary conditions along the other edges are considered. To illustrate the new model, both uniaxial and biaxial loads are applied and the critical buckling loads are defined for over a wide range of thickness, different length scale parameters and various boundary conditions. To show the accuracy of the formulations, present results are compared with available results in literature for specific cases and a very good agreement is observed. Results reveal that the critical buckling load increases as the length scale parameter increases especially when the thickness of the micro-plates becomes in order of length scale parameter and this effect is more significant for free boundary condition.
\end{abstract}

Key words: Micro-plate / Levy solution / buckling / couple stress

\section{Introduction}

Micro and nano structures such as micro-beams and micro-plates are extensively used in micro and nano electro-mechanical systems (MEMS and NEMS) [1,2]. Atomic and molecular models can be used to study the mechanical analysis of micro and nano structures; however, they are more complex and expensive than continuum models, which can provide a much simpler approach. In the classical continuum theories as an assumption that material distribution is continuously throughout the body, mechanical behaviors of materials could not be precisely predicted due to the lack of material size-effects. The small length scale theories such as nonlocal elasticity theory $[3,4]$, surface elasticity [5] and couple stress theory $[6,7]$ have been developed to eliminate the lack of material size-effects in classical theories. In the classical couple stress theory two separate material length scale parameters exist and it is a very difficult task to experimentally determine two micro-structural material length scale parameters. Due to this problem, Yang et al. [8] proposed the modified couple stress theory in which the strain energy is proposed to be a quadratic function of

\footnotetext{
${ }^{a}$ Corresponding author: e.jomehzadeh@kgut.ac.ir
}

the strain tensor and the symmetric part of the curvature tensor, and only one length scale parameter is included. In the other word, the modified couple stress theory contains only one length scale parameter, which can capture size effects at the micro scale.

Due to the wide application of micro structures, many researchers performed non-classical theories to describe mechanical behaviors of materials. When it comes to micro-beam analysis, Park and Gao [9] developed a model for bending of a Bernoulli-Euler beam by using a modified couple stress theory. The dynamic behaviors of simply supported Bernoulli-Euler beams were investigated by Kong et al. [10] on the basis of the modified couple stress theory. Bending of a functionally graded microscale Timoshenko beam was studied by Simsek et al. [11]. They analytically solved governing equations of a simply supported micro-beam subjected to a point and uniformly distributed loads. Nateghi et al. [12] studied the buckling analysis of functionally graded micro-beams by considering classical, first and third order shear deformation beam theories. Akgoz and Civalek [13] investigated free vibration analysis of an axially functionally graded tapered Bernoulli-Euler micro-beam which was considered to be fixed at one end and free at the other edges. Roque et al. [14] employed modified couple stress theory 
to study the bending of simply supported laminated composite micro-beams subjected to transverse loads by using meshless numerical method. Ke et al. [15] investigated nonlinear free vibration of micro-beams made of functionally graded materials using von Kármán geometric nonlinearity.

As far as micro-plates analysis is concerned, Akgoz and Civalek [16] studied free vibration analysis of singlelayered graphene sheets in an elastic matrix via modified couple stress theory. In addition, they presented analytical solution for bending, buckling and vibration of micro-sized plates resting on an elastic medium by employing Navier's method [17]. The derivation of the governing equations of motion and corresponding boundary conditions for geometrically nonlinear micro-plates with arbitrary shapes based on the modified couple stress theory was obtained by Asghari [18]. A general thin plate theory including surface effects was proposed by $\mathrm{Lu}$ et al. [19] for static and dynamic analyses of plate like thin film structures. Lim and He [20] studied the sizedependent geometrically nonlinear response of thin elastic films with nano-scale thickness based on a continuum approach. The film was assumed elastically isotropic, and the Kirchhoff's hypothesis was adopted to approximate the kinetics relations. Jomehzadeh et al. [21] investigated the vibration analysis of micro-plates based on the modified couple stress theory. Reddy and Kim [22] considered nonlinear modified couple stress theory based on the third-order theory for functionally graded micro-plates. Size-dependent model for free vibration and bending for functionally graded Reddy plate was developed by Thai and Kim [23]. They used modified couple stress theory to capture small scale effects. Also, Thai and Choi [24] studied buckling and vibration responses of simply supported micro-plates based on modified couple stress theory.

Also, some authors used nonlocal and strain gradient theories to study the mechanical behaviour of nano structures. Free vibration analysis of microtubules was investigated by Civalek and Akgoz [25] based on the nonlocal Euler-Bernoulli beam theory. Buckling analysis of biaxially compressed graphene sheets with nonlocal elasticity theory was studied by Pradhan [26] based on the Levytype solution. Akgoz and Civalek [27] presented bending analysis of micro-sized beams based on the modified strain gradient and couple stress theories.

To the best of author knowledge, there is no work in literature that investigates the effect of different boundary conditions on the buckling behavior of micro-plates analytically. In the present work, buckling analysis of micro plates subjected to uniaxial and biaxial loads is developed by considering small scale effect. A Levy solution is employed to predict critical buckling loads for different boundary conditions. The buckling loads are analytically defined and the effects of boundary conditions on buckling behaviors of micro-plates are studied for the first time. It is found that the effect of small length scale on increasing of stiffness is more considerable for micro-plates with free boundary condition.

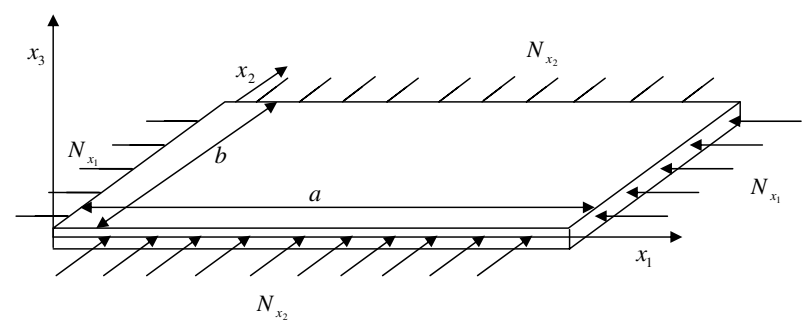

Fig. 1. Geometry of a rectangular micro-plate.

\section{The modified couple stress formulations}

The linear theory of elasticity is based on the assumption that only the symmetric strain tensor is responsible for strain energy and stress in the body. This theory could not display mechanical behavior of micro and nano structures since the skew-symmetric part of the displacement gradient which represents a rigid body rotation of the material elements is not considered. In the modified couple stress theory, the strain energy density includes both strain and gradient of the rotation vector as [8]

$$
U=1 / 2 \int_{V}\left(\sigma_{i j} \varepsilon_{i j}+m_{i j} \chi_{i j}\right) d V
$$

where $\sigma_{i j}, \varepsilon_{i j}, m_{i j}$ and $\chi_{i j}$ are the Cauchy stress, Green strain, deviatoric part of couple stress and symmetric curvature tensor respectively, which are defined as

$$
\begin{aligned}
\sigma_{i j} & =\lambda \varepsilon_{k k} \delta_{i j}+2 \mu \varepsilon_{i j} \\
\varepsilon_{i j} & =1 / 2\left(u_{i, j}+u_{j, i}\right) \\
m_{i j} & =2 \mu l^{2} \chi_{i j} \\
\chi_{i j} & =1 / 2\left(\theta_{i, j}+\theta_{j, i}\right)
\end{aligned}
$$

where $\lambda$ and $\mu$ are the Lame's coefficients and $l$ is a length scale parameter and mathematically is the square root of the ratio of the modulus of curvature to the shear modulus [8]. This length scale parameter is a material property and contains the difference between classical and modified couple stress elasticity theories. This parameter is small with respect to dimensions of the structure, and its influence becomes important as dimensions of a body diminish to the order of the length scale parameter. The parameter $\theta_{i}$ in equation $(2 \mathrm{~d})$ is the rotation vector and can be expressed in term of displacement vector as

$$
\theta_{i}=1 / 2 e_{i j k} u_{k, j}
$$

where $e_{i j k}$ is the permutation symbol.

\section{Stability equations of micro-plates}

Let us consider a rectangular micro-plate with dimension $a \times b$ and uniform thickness $h$ (Fig. 1). The displacement field of such plate is assumed to be

$$
\begin{aligned}
& u_{1}\left(x_{1}, x_{2}, x_{3}\right)=u_{0}\left(x_{1}, x_{2}\right)+x_{3} \psi_{1}\left(x_{1}, x_{2}\right) \\
& u_{2}\left(x_{1}, x_{2}, x_{3}\right)=v_{0}\left(x_{1}, x_{2}\right)+x_{3} \psi_{2}\left(x_{1}, x_{2}\right) \\
& u_{3}\left(x_{1}, x_{2}, x_{3}\right)=w\left(x_{1}, x_{2}\right)
\end{aligned}
$$


where $u_{0}, v_{0}$ and $w$ are the displacement components of the middle surface in $x_{1}, x_{2}$ and $x_{3}$ directions, respectively, $\psi_{1}$ and $\psi_{2}$ are considered as the rotation functions of the middle surface and based on the Kirchhoff assumption, they are defined as [28]

$$
\begin{aligned}
& \psi_{1}=-w_{, 1} \\
& \psi_{2}=-w_{, 2}
\end{aligned}
$$

where subscript comma denotes derivation with respect to independent variables. Under the assumption of large deformation with Von-Karman assumption [29] the nonlinear strain components can be expressed as

$$
\begin{aligned}
& \varepsilon_{11}=u_{0,1}-x_{3} w_{, 11}+w_{, 1}^{2} / 2 \\
& \varepsilon_{12}=\varepsilon_{21}=1 / 2\left(u_{0,2}+v_{0,1}-2 x_{3} w_{, 12}+w_{, 1} w_{, 2}\right) \\
& \varepsilon_{22}=v_{0,2}-x_{3} w_{, 22}+w_{, 2}^{2} / 2
\end{aligned}
$$

In addition, the rotation and curvature components are obtained as

$$
\begin{aligned}
\theta_{1} & =w_{, 2}, \quad \theta_{2}=-w_{, 1}, \quad \theta_{3}=\left(v_{0,1}-u_{0,2}\right) / 2 \\
\chi_{11} & =w_{, 12}, \quad \chi_{12}=\chi_{21}=1 / 2\left(w_{, 22}-w_{, 11}\right) \\
\chi_{13} & =\chi_{31}=1 / 4\left(v_{0,11}-u_{0,12}\right) \\
\chi_{22} & =-w_{, 12}, \quad \chi_{23}=\chi_{32}=1 / 4\left(v_{0,12}-u_{0,22}\right), \chi_{33}=0
\end{aligned}
$$

The equilibrium equations and corresponding boundary conditions can be obtained by using the theory of minimum potential energy, i.e.

$$
\Pi=\int_{0}^{t}((\delta U+\delta \Omega)) d t
$$

where $U$ and $\Omega$ are the strain energy and potential energy of the transverse loads applied to the edges of the micro-plate, respectively. Considering the couple stress effect, the strain energy involves both classical and couple stresses terms as shown in equation (1). Expressing the strain and symmetric curvature tensors in terms of displacement components, the variation of the strain energy density of the micro-plate can be obtained as

$$
\begin{aligned}
\delta U= & \int_{V}\left[\sigma_{11}\left(\delta u_{0,1}-x_{3} \delta w_{, 11}+w_{, 1} \delta w_{, 1}\right)\right. \\
& +\sigma_{22}\left(\delta v_{0,2}-x_{3} \delta w_{, 22}+w_{, 2} \delta w_{, 2}\right) \\
& \left.+\sigma_{12}\left(\delta u_{0,2}+\delta v_{0,1}-2 x_{3} \delta w_{, 12}\right)\right] d V \\
& +\int_{V}\left[\left(m_{11}-m_{22}\right) \delta w_{, 12}+m_{12}\left(\delta w_{, 22}-\delta w_{, 11}\right)\right. \\
& \left.+m_{13}\left(\delta v_{0,11}-\delta u_{0,12}\right) / 2+m_{23}\left(\delta v_{0,12}-\delta u_{0,22}\right) / 2\right] d V
\end{aligned}
$$

also, the work done by transverse forces can be expressed as

$$
\delta \Omega=-\int_{A} p \delta w d A
$$

Introducing the equations (9) and (10) into the theory of minimum potential energy, the equilibrium equations for a micro-plate can be obtained as

$$
\begin{aligned}
& N_{11,1}+N_{12,2}+1 / 2 Y_{13,12}+1 / 2 Y_{23,22}=0 \\
& N_{12,1}+N_{22,2}-1 / 2 Y_{13,11}-1 / 2 Y_{23,12}=0 \\
& M_{11,11}+2 M_{12,12}+M_{22,22}-Y_{11,12}-Y_{12,22} \\
& +Y_{12,11}+Y_{22,12}+N_{x} w_{, 11}+N_{y} w_{, 22}=0
\end{aligned}
$$

where $N_{x}$ and $N_{y}$ are lateral forces in $x_{1}$ and $x_{2}$ directions, respectively.

In addition, the corresponding boundary conditions are

$$
\begin{aligned}
\delta u= & 0 \text { or }\left(N_{11}+1 / 4 Y_{13,2}\right) n_{1} \\
& +\left(N_{12}+1 / 4 Y_{13,1}+1 / 2 Y_{23,2}\right) n_{2}=0 \\
\delta u_{, 1}= & 0 \text { or }\left(-1 / 4 Y_{13}\right) n_{2}=0 \\
\delta u_{, 2}= & 0 \text { or }\left(-1 / 4 Y_{13}\right) n_{1}+\left(-1 / 2 Y_{23}\right) n_{2}=0 \\
\delta v= & 0 \text { or }\left(N_{12}-1 / 2 Y_{13,1}-1 / 4 Y_{23,2}\right) n_{1} \\
& +\left(N_{22}-1 / 4 Y_{23,1}\right) n_{2}=0 \\
\delta v_{, 1}= & 0 \text { or }\left(1 / 2 Y_{13}\right) n_{1}+\left(1 / 4 Y_{23}\right) n_{2}=0 \\
\delta v_{, 2}= & 0 \text { or }\left(1 / 4 Y_{23}\right) n_{1}=0 \\
\delta w= & 0 \text { or }\left(M_{11,1}+M_{12,2}-1 / 2 Y_{11,2}+Y_{12,1}\right. \\
& \left.+1 / 2 Y_{22,2}\right) n_{1}+\left(M_{12,1}+M_{22,2}-1 / 2 Y_{11,1}\right. \\
& \left.-Y_{12,2}+1 / 2 Y_{22,1}\right) n_{2}=0 \\
\delta w_{, 1}= & 0 \text { or }\left(-M_{11}-Y_{12}\right) n_{1} \\
& +\left(-M_{12}+1 / 2 Y_{11}-1 / 2 Y_{22}\right) n_{2}=0 \\
\delta w_{, 2}= & 0 \text { or }\left(-M_{12}+1 / 2 Y_{11}-1 / 2 Y_{22}\right) n_{1} \\
& +\left(-M_{22}+Y_{12}\right) n_{2}=0
\end{aligned}
$$

Also, resultant parameters $\left(N_{i j}, M_{i j}, Y_{i j}\right)$ can be defined by integrating corresponding stresses along the thickness as follows

$$
\begin{aligned}
N_{i j} & =\int_{-h / 2}^{h / 2} \sigma_{i j} d x_{3} \\
M_{i j} & =\int_{-h / 2}^{h / 2} \sigma_{i j} x_{3} d x_{3} \\
Y_{i j} & =\int_{-h / 2}^{h / 2} m_{i j} d x_{3}
\end{aligned}
$$

By the above equations, the resultant parameters can be easily defined in terms of displacement components. By substituting the results into the equilibrium equations (11), the governing equations can be determined. Using adjacent-equilibrium criteria [30], the governing 
stability equations of micro-plate are obtained as

$$
\begin{aligned}
& \frac{E h}{\left(1-\nu^{2}\right)}\left\{u_{0,11}+\frac{1-\nu}{2} u_{0,22}+\frac{1+\nu}{2} v_{0,12}\right. \\
& \left.\quad+\frac{l^{2}(1-\nu)}{8}\left(v_{0,1112}+v_{0,1222}-u_{0,1122}-u_{0,2222}\right)\right\}=0
\end{aligned}
$$

$$
\begin{aligned}
& \frac{E h}{\left(1-\nu^{2}\right)}\left\{v_{0,22}+\frac{1-\nu}{2} v_{0,11}+\frac{1+\nu}{2} u_{0,12}\right. \\
& \left.\quad+\frac{l^{2}(1-\nu)}{8}\left(u_{0,1112}+u_{0,1222}-v_{0,1122}-v_{0,1111}\right)\right\}=0
\end{aligned}
$$

$$
\begin{aligned}
-\left(D+\mu h l^{2}\right)\left(w_{, 1111}+2 w_{, 1122}+w_{, 2222}\right) & \\
& +N_{x} w_{, 11}+N_{y} w_{, 22}=0
\end{aligned}
$$

where $E$ and $\nu$ are the Young modulus and Poisson ratio of the micro-plate and $D$ is the classical flexural stiffness as $E h^{3} / 12\left(1-\nu^{2}\right)$. Unlike the classical Kirchhoff plate theory, it can be seen that the current model based on the modified couple stress theory contains a material length scale parameter $l$, which can capture the size effect. Also it can be concluded that due to presence of material length scale parameter, the stiffness of the micro-plate model $\left(D+\mu h l^{2}\right)$ is more than the stiffness of classical plate $(D)$.

\section{Buckling analysis}

For symmetrically and homogeneous micro-plates, the first two stability equations (14a) and (14b) are uncoupled from the last stability equation. Therefore, for buckling analysis only the third equilibrium equation is needed to be considered. We consider a micro-plate that is only simply supported at two opposite edges $x_{1}=0$ and $x_{1}=a$ with the following boundary conditions:

$$
\begin{array}{ll}
w\left(0, x_{2}\right)=0, & M_{11}\left(0, x_{2}\right)+Y_{12}\left(0, x_{2}\right)=0 \\
w\left(a, x_{2}\right)=0, & M_{11}\left(a, x_{2}\right)+Y_{12}\left(a, x_{2}\right)=0
\end{array}
$$

It is considered that the micro-plates can have three different boundary conditions along the other edges: simply, clamp or free. Based on the Levy solution, the transverse displacement function is assumed as

$$
w\left(x_{1}, x_{2}\right)=\sum_{m=1}^{\infty} w_{m}\left(x_{2}\right) \sin \left(m \pi x_{1} / a\right)
$$

where $m$ is the number of half waves in $x_{1}$ direction. It can be easily found that the following series solution exactly satisfies the simply supported boundary conditions (15) in $x_{1}$ direction. By substituting relation (16) into the third governing stability equation (14c), an ordinary partial differential equation in term of transverse deflection can be obtained. Solving the resulting differential equation yields

$$
\begin{aligned}
w_{m}\left(x_{2}\right)= & C_{1} \sinh \left(\frac{\sqrt{-B+\sqrt{B^{2}-4 A C}}}{2 A} x_{2}\right) \\
& +C_{2} \cosh \left(\frac{\sqrt{-B+\sqrt{B^{2}-4 A C}}}{2 A} x_{2}\right) \\
& +C_{3} \sinh \left(\frac{\sqrt{-B-\sqrt{B^{2}-4 A C}}}{2 A} x_{2}\right) \\
& +C_{4} \cosh \left(\frac{\sqrt{-B-\sqrt{B^{2}-4 A C}}}{2 A} x_{2}\right)
\end{aligned}
$$

where the parameters $A, B$ and $C$ are defined in terms of physical properties of the micro-plate and lateral forces as

$$
\begin{aligned}
& A=-D-\mu h l^{2} \\
& B=2\left(D+\mu h l^{2}\right) \beta_{m}^{2}-N_{y} \\
& C=-\left(D+\mu h l^{2}\right) \beta_{m}^{4}+N_{x} \beta_{m}^{2}
\end{aligned}
$$

This solution is valid for $B^{2}-4 A C>0$. However, in the case of $B^{2}-4 A C<0$, the solution is in the following form

$$
\begin{aligned}
w_{m}(x)= & C_{1} \cosh (s y) \cos (t y)+C_{2} \sinh (s y) \sin (t y) \\
& +C_{3} \sinh (s y) \cos (t y)+C_{4} \cosh (s y) \sin (t y)
\end{aligned}
$$

where $s$ and $t$ are real and imaginary part of $(-B-$ $\left.\sqrt{B^{2}-4 A C}\right) / 2 A$, respectively.

To determine the critical buckling loads, the arbitrary boundary conditions along the other edges at $x_{2}=0$ and $x_{2}=b$ should be imposed. The conditions can be written as

$$
\begin{aligned}
& \text { Simply supported }(\mathbf{S}): w=-M_{22}+Y_{12}=0 \\
& \begin{array}{r}
\text { Clamped }(\mathbf{C}): w=w_{, 2}=0 \\
\text { Free }(\mathbf{F}):-M_{22}+Y_{12}=M_{22,2}+2 M_{12,1}-Y_{11,1} \\
\quad-Y_{12,2}+Y_{22,1}-N_{x} w_{, 2}=0
\end{array}
\end{aligned}
$$

It can be seen that only the clamped boundary condition does not explicitly depend on the small scale effects. The critical buckling load of the micro-plate can be evaluated by applying these conditions and setting the determinant of the fourth order coefficient matrix equal to zero.

\section{Numerical results and discussion}

In order to find accuracy of the present formulations, a comparison has been carried out with critical buckling loads of classical rectangular plates $(l=0)$. Non-dimensional buckling forces $\bar{P}=N a^{2} / D$ for biaxial $\operatorname{case}\left(N=N_{x}=N_{y}\right)$ are compared with $\mathrm{Yu}$ and Wang [31] in Table 1. It can be found that the present 
Table 1. Comparison of the non-dimensional biaxial buckling load $\bar{P}$ of classical plates $(l=0, v=0.3)$.

\begin{tabular}{ccccccc}
\hline \multirow{2}{*}{$a / b$} & \multicolumn{2}{c}{ SSSS } & \multicolumn{2}{c}{ SSCC } & \multicolumn{2}{c}{ SSFF } \\
\cline { 2 - 7 } & Ref. [31] & Present & Ref. [31] & Present & Ref. [31] & Present \\
\hline 5 & 256.61 & 256.6097 & 927.99 & 927.4107 & 8.994 & 8.9953 \\
2 & 49.35 & 49.3480 & 150.99 & 150.9919 & 9.060 & 9.0583 \\
1 & 19.74 & 19.7392 & 37.80 & 37.7996 & 9.199 & 9.2005 \\
0.5 & 12.33 & 12.3370 & 14.62 & 14.6174 & 9.400 & 9.4007 \\
0.1 & 9.967 & 9.9683 & 9.979 & 9.9821 & 9.629 & 9.6264 \\
\hline
\end{tabular}

Table 2. Accurate uniaxial buckling loads of micro-plates in $x_{1}$ direction.

\begin{tabular}{cccccc}
\hline $\begin{array}{c}\text { Boundary } \\
\text { conditions }\end{array}$ & $a / b$ & $l / h=0$ & $l / h=0.5$ & $l / h=1$ & $l / h=5$ \\
\hline \multirow{2}{*}{ SSSS } & 0.5 & 23.3590 & 45.0828 & 110.2544 & 2195.7447 \\
& 1 & 14.9497 & 28.8530 & 70.5628 & 1405.2766 \\
& 1.5 & $16.2215(2)$ & $31.3075(2)$ & $76.5656(2)$ & $1524.8227(2)$ \\
\hline \multirow{3}{*}{$\mathrm{SSSC}$} & 0.5 & 25.6132 & 49.4334 & 120.8941 & 2407.6377 \\
& 1 & 21.4537 & 41.4056 & 101.2613 & 2016.6450 \\
& 1.5 & $20.2988(2)$ & $39.1766(2)$ & $95.8101(2)$ & $1908.0833(2)$ \\
\hline & 0.5 & 28.7456 & 55.4792 & 135.6797 & 2702.0953 \\
$\mathrm{SSCC}$ & 1 & $28.7456(2)$ & $55.4792(2)$ & $135.6797(2)$ & $2702.0953(2)$ \\
& 1.5 & $26.5953(2)$ & $51.3290(2)$ & $125.5300(2)$ & $2499.9625(2)$ \\
\hline & 0.5 & 15.8900 & 33.7868 & 88.4911 & 1834.4649 \\
$\mathrm{SSSF}$ & 1 & 5.0021 & 11.3854 & 31.5905 & 683.4221 \\
& 1.5 & 3.0016 & 7.1044 & 20.1494 & 442.5192 \\
\hline & 0.5 & 14.2412 & 30.2842 & 79.9501 & 1670.7807 \\
$\mathrm{SSFF}$ & 1 & 3.4347 & 7.8790 & 22.5457 & 498.6528 \\
& 1.5 & 1.4894 & 3.6144 & 10.8576 & 248.7279 \\
\hline & 0.5 & 16.2413 & 34.8605 & 92.0960 & 1921.1542 \\
$\mathrm{SSCF}$ & 1 & 5.8551 & 13.5059 & 37.7515 & 821.0607 \\
& 1.5 & 4.5118 & 10.5527 & 29.5299 & 642.8876 \\
\hline
\end{tabular}

results are in good agreement with the available results in the literature.

The critical buckling loads of micro-plates are presented in Tables 2-4 for different boundary conditions, several length scale ratios and various dimensions. The boundary conditions are identified according to the conditions along the $x_{1}$ and $x_{2}$ directions (e.g. SSCF denotes two edges simply supported along the $x_{1}$ edges and Clamped and Free edges along the $x_{2}$ edges). In this model, material properties of the micro-plate are considered as $\nu=0.38$ and $E=1.44 \mathrm{GPa}$. Also, the number in parenthesis indicates the number of buckling mode shapes. It can be seen that for all values of length scale parameters, the critical buckling load increases as length scale ratio increases and for some boundary conditions in specific aspect ratios, the critical buckling load occurs in second mode. In case of uniaxial buckling load for a micro plate with all edges simply supported, it can be seen that when the in-plane load is in $x$ direction, the unity aspect ratio is a turning point. In the other words, the buckling load decreases for aspect ratios up to unity and after that it increases. The most striking feature is the effect of boundary conditions on the critical buckling loads of micro-plates. Length scale parameter appears in boundary conditions of free and simply supported edges and this causes micro-plate became more stiff as the length scale parameter increases. This effect is more evident for case free since its boundary conditions are more reliant on length scale parameter compared to other boundary conditions.

The effect of length scale, also, on higher buckling modes can be studied. The values of buckling loads are presented for different modes in Table 5 . It can be seen that the length scale effect is more significant for higher buckling modes.

In order to demonstrate the effect of length scale parameter on buckling behavior of micro-plates, the critical biaxial buckling loads of square micro-plates are depicted in Figure 2 for three boundary conditions along $x_{2}$ direction with $a=b=50 h$. Different values for material length scale parameter are considered in these figures. It can be seen how the fundamental buckling loads predicted by the modified couple stress models change with thickness of the micro-plate for different boundary conditions. For all boundary conditions, significant effects due to the length scale parameter can be observed. It can be seen that the fundamental buckling loads predicted by the new model are higher than those predicted by the classical Kirchhoff plate theory especially in low values of $h$. This is due to the increased stiffness predicts by the present model. Also, it can be found that the difference in buckling loads between classical and present model is more significant when the thickness of the micro-plate is small with respect to the length scale parameter, while it is decreasing 
Table 3. Accurate uniaxial buckling loads of micro-plates in $x_{2}$ direction.

\begin{tabular}{cccccc}
\hline $\begin{array}{c}\text { Boundary } \\
\text { conditions }\end{array}$ & $a / b$ & $l / h=0$ & $l / h=0.5$ & $l / h=1$ & $l / h=5$ \\
\hline \multirow{2}{*}{ SSSS } & 0.5 & 59.7999 & 115.4121 & 282.2513 & 5621.1025 \\
& 1 & 14.9497 & 28.8530 & 70.5628 & 1405.2766 \\
& 1.5 & 6.6443 & 12.8236 & 31.3613 & 624.5674 \\
\hline \multirow{2}{*}{ SSSC } & 0.5 & 63.3374 & 122.2412 & 298.9526 & 5953.7179 \\
& 1 & 18.1159 & 34.9637 & 85.5071 & 1702.8964 \\
& 1.5 & 11.5677 & 22.3256 & 54.5995 & 1087.3638 \\
\hline \multirow{3}{*}{ SSCC } & 0.5 & 72.4637 & 139.8549 & 342.0285 & 6811.5855 \\
& 1 & 25.2022 & 48.6403 & 118.9546 & 2369.0118 \\
& 1.5 & 18.8246 & 36.3316 & 88.8523 & 1769.5169 \\
\hline \multirow{3}{*}{ SSSF } & 0.5 & 31.3260 & 80.8609 & 245.1127 & 5592.4818 \\
& 1 & 7.9997 & 20.8500 & 62.7500 & 1398.3386 \\
& 1.5 & 3,4437 & 9.4934 & 31.3612 & 624.5674 \\
\hline \multirow{2}{*}{ SSFF } & 0.5 & 30.7333 & 78.4480 & 235.8013 & 5545.6189 \\
& 1 & 6.8337 & 18.0664 & 56.4648 & 1385.3091 \\
& 1.5 & 2.5465 & 6.5043 & 19.0312 & 426.1901 \\
\hline \multirow{2}{*}{ SSCF } & 0.5 & 31.3327 & 80.8321 & 245.0286 & 5621.1065 \\
& 1 & 8.0498 & 21.3049 & 66.9430 & 1405.2766 \\
\hline
\end{tabular}

Table 4. Accurate biaxial buckling loads of micro-plates.

\begin{tabular}{cccccc}
\hline $\begin{array}{c}\text { Boundary } \\
\text { conditions }\end{array}$ & $a / b$ & $l / h=0$ & $l / h=0.5$ & $l / h=1$ & $l / h=5$ \\
\hline \multirow{2}{*}{ SSSS } & 0.5 & 18.6872 & 36.0663 & 88.2035 & 1756.5958 \\
& 1 & 7.4749 & 14.4265 & 35.2814 & 702.6383 \\
& 1.5 & 5.3985 & 10.4191 & 25.4810 & 507.4610 \\
\hline \multirow{2}{*}{ SSSC } & 0.5 & 20.0509 & 38.6982 & 94.6403 & 1884.7862 \\
& 1 & 9.9517 & 19.2067 & 46.9718 & 935.4559 \\
& 1.5 & 8.4739 & 16.3547 & 39.9970 & 796.5509 \\
\hline \multirow{3}{*}{ SSCC } & 0.5 & 22.1414 & 42.7329 & 104.5075 & 2081.2934 \\
& 1 & 14.3140 & 27.6261 & 67.5622 & 1345.5175 \\
& 1.5 & 14.0811 & 27.1765 & 66.4628 & 1323.6231 \\
\hline \multirow{3}{*}{ SSSF } & 0.5 & 14.0628 & 32.6155 & 86.0757 & 1755.0007 \\
& 1 & 3.6634 & 9.1654 & 27.8868 & 677.4980 \\
& 1.5 & 1.7141 & 4.3603 & 13.2355 & 309.4420 \\
\hline \multirow{2}{*}{ SSFF } & 0.5 & 13.6170 & 31.2646 & 84.3756 & 1753.4065 \\
& 1 & 3.3044 & 8.2525 & 25.9833 & 666.9084 \\
& 1.5 & 1.4477 & 3.7441 & 12.2161 & 307.7936 \\
\hline \multirow{2}{*}{ SSCF } & 0.5 & 21.2694 & 33.4978 & 91.1971 & 1883.8700 \\
& 1 & 3.9299 & 10.0088 & 31.0033 & 794.9042 \\
\hline
\end{tabular}

or even diminishing with increasing of the thickness. This reveals that the size effect is considerable only when the plate thickness is as small as the material length scale parameter.

The critical buckling load is depicted for two values of length scale parameters in Figure 3. It can be seen that the variation of buckling load with respect to the thickness is linear for classical theory $(l=0)$. However, for couple stress theory, this variation is completely nonlinear and it has a descending behavior.
Table 5. Higher uniaxial buckling loads of SSCC micro-plates in $x_{2}$ direction.

\begin{tabular}{ccccc}
\hline Mode no. & $l / h=0$ & $l / h=0.5$ & $l / h=1$ & $l / h=5$ \\
\hline 1 & 52.2022 & 48.6403 & 118.9546 & 2369.0118 \\
2 & 38.8188 & 74.9203 & 183.2248 & 3648.9694 \\
3 & 67.9931 & 131.2266 & 320.9272 & 9224.3136 \\
4 & 98.1400 & 189.3928 & 463.1783 & 13379.8073 \\
\hline
\end{tabular}

In order to obtain the effect of boundary conditions on buckling behavior of micro-plates, the critical 


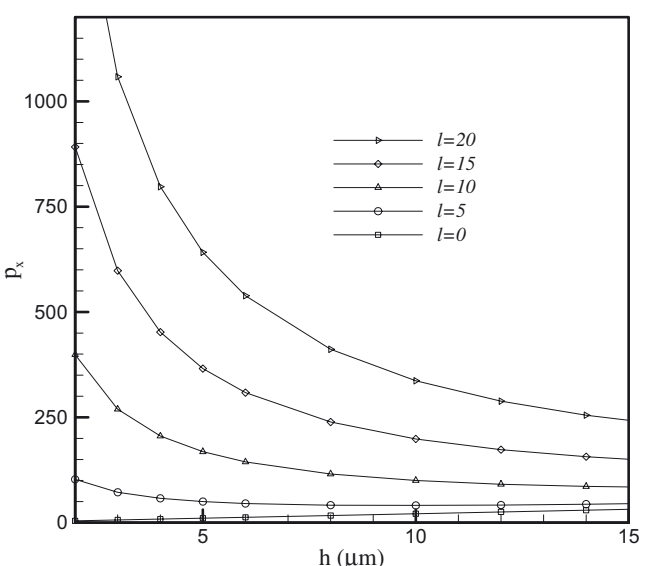

(a)

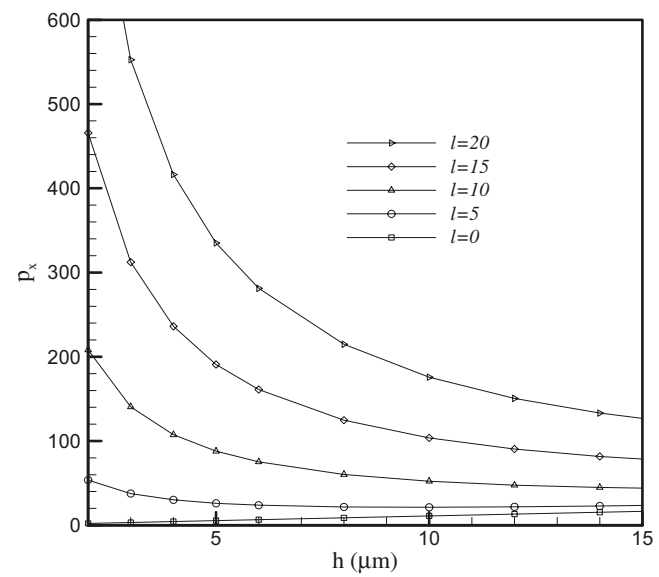

(b)

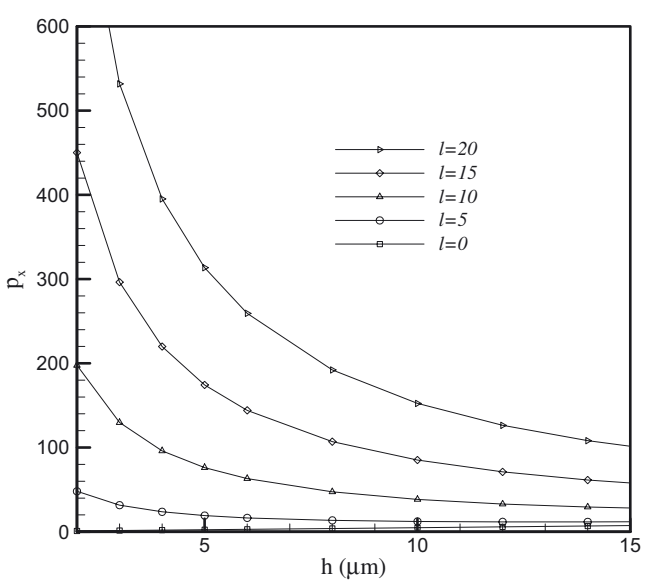

(c)

Fig. 2. Variation of critical biaxial buckling loads versus the thickness of the micro-plate (a) SSCC, (b) SSSS, (c) SSFF.

buckling load is shown in Figure 4 for different boundary conditions. It can be concluded the critical buckling load decreases with increasing of thickness for its lower values. For higher values for thickness, the ascending behavior is seen for some boundary conditions.

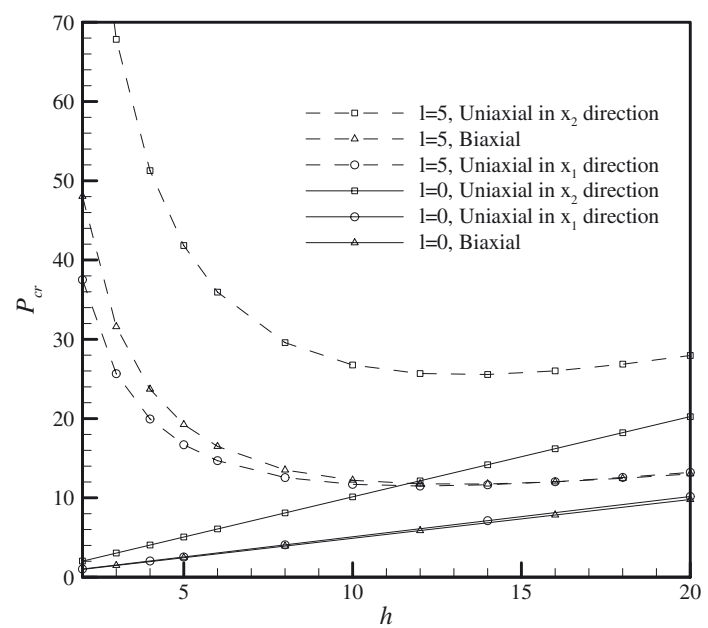

Fig. 3. Variation of critical buckling load of SSFF mirco-plates.

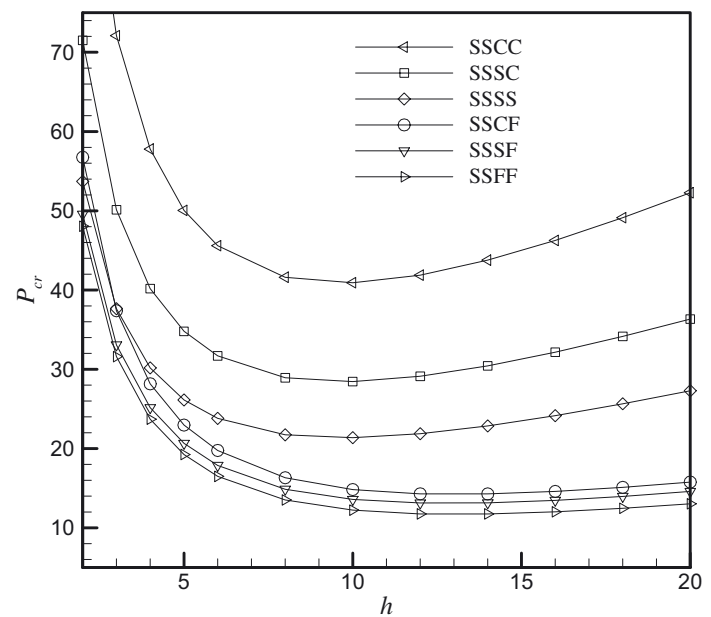

Fig. 4. Variation of critical biaxial buckling load for different boundary conditions of micro-plates $(l=5 \mu \mathrm{m})$.

\section{Conclusion}

A Levy solution has been presented for buckling analysis of micro-plates based on the modified couple stress theory. This model contains only one additional material constant and can explain the size effect of microstructures. Using the theory of minimum potential energy and adjacent equilibrium criteria, the stability equations have been obtained for thin micro-plates. The governing stability equation has been solved for a homogeneous isotropic micro-plate with two opposite edges simply supported and arbitrary boundary conditions along the other edges. The buckling loads have been presented for a wide range of length scale parameters, different boundary conditions and some thickness ratios. It was found that a behavior more complex than classical plate model emerges. It has been concluded that the critical buckling loads predicted by the new model are higher than those predicted by the classical Kirchhoff plate theory. The effects of boundary conditions on the buckling of micro-plates have 
been clearly explained and it was found that small scale effect is more significant for free boundary conditions.

\section{References}

[1] X. Li, B. Bhushan, K. Takashima, C.W. Baek, Y.K. Kim, Mechanical characterization of micro/nanoscale structures for MEMS/NEMS applications using nanoindentation techniques, Ultramicroscopy 97 (2003) 481-494

[2] J. Pei, F. Tian, T. Thundat, Glucose biosensor based on the microcantilever, Anal. Chem. 76 (2004) 292-297

[3] A.C. Eringen, On differential equations of nonlocal elasticity and solutions of screw dislocation and surface waves, J. Appl. Phys. 54 (1983) 4703-4710

[4] A.C. Eringen, Edelen DGB On nonlocal elasticity, Int. J. Eng. Sci. 10 (1972) 233-248

[5] A. Khajeansari, G.H. Baradaran, Surface effect investigation for static bending of nanowires resting on elastic substrate using Timoshenko beam theory in tandem with the Laplace-Young equation, Mechanics \& Industry 13 (2012) 163-174

[6] R.A. Toupin, Elastic materials with couple-stresses, Arch. Ration. Mech. Anal. 11 (1962) 385-414

[7] R.D. Mindlin, H.F. Tiersten, Effects of couple-stresses in linear elasticity, Arch. Ration. Mech. Anal. 11 (1962) 415-448

[8] F. Yang, A.C.M. Chong, D.C.C. Lam, P. Tong, Couple stress based strain gradient theory for elasticity, Int. J. Sol. Struct. 39 (2002) 2731-2743

[9] S.K. Park, X.L. Gao, Bernoulli-Euler beam model based on a modified couple stress theory, J. Micromech. Microeng. 16 (2006) 2355-2359

[10] S. Kong, S. Zhou, Z. Nie, K. Wang, The size-dependent natural frequency of Bernoulli-Euler micro-beams, Int. J. Eng. Sci. 46 (2008) 427-437

[11] M. Simsek, T. Kocatürk, S.D. Akbas, Static bending of a functionally graded microscale Timoshenko beam based on the modified couple stress theory, Compos. Struct. 95 (2013) $740-747$

[12] A. Nateghi, M. Salamat-talab, J. Rezapour, B. Daneshian, Size dependent buckling analysis of functionally graded micro beams based on modified couple stress theory, Appl. Math. Model. 36 (2012) 4971-4987

[13] B. Akgoz, O. Civalek, Free vibration analysis axially functionally graded tapered Bernoulli-Euler microbeams based on the modified couple stress theory, Compos. Struct. 98 (2013) 314-322

[14] C.M.C. Roque, D.S. Fidalgo, A.J.M. Ferreira, J.N. Reddy, A study of a microstructure-dependent composite laminated Timoshenko beam using a modified couple stress theory and a meshless method, Compos. Struct. 96 (2013) 532-537
[15] L.L. Ke, Y. Wang, J. Yang, S. Kitipornchai, Nonlinear free vibration of size-dependent functionally graded microbeams, Int. J. Eng. Sci. 50 (2012) 256-267

[16] B. Akgoz, O. Civalek, Free vibration analysis for singlelayered graphene sheets in an elastic matrix via modified couple stress theory, Mater. Design 42 (2012) 164-171

[17] B. Akgoz, O. Civalek, Modeling and analysis of microsized plates resting on elastic medium using the modified couple stress theory, Meccanica 48 (2013) 863-873

[18] M. Asghari, Geometrically nonlinear micro-plate formulation based on the modified couple stress theory, Int. J. Eng. Sci. 51 (2012) 292-309

[19] P. Lu, L.H. He, H.P. Lee, C. Lu, Thin plate theory including surface effects, Int. J. Solid. Struct. 43 (2006) 4631-4647

[20] C.W. Lim, L.H. He, Size-dependent nonlinear response of thin elastic films with nano-scale thickness, Int. J. Mech. Sci. 46 (2004) 1715-1726

[21] E. Jomehzadeh, H.R. Noori, A.R. Saidi, The sizedependent vibration analysis of micro-plates based on a modified couple stress theory, Phys. E 43 (2011) 877-883

[22] J.N. Reddy, J. Kim, A nonlinear modified couple stressbased third-order theory of functionally graded plates, Compos. Struct. 94 (2012) 1128-1143

[23] H.T. Thai, S.E. Kim, A size-dependent functionally graded Reddy plate model based on a modified couple stress theory, Composites B: Eng. 45 (2013) 1636-1645

[24] H.T. Thai, D.H. Choi, Size-dependent functionally graded Kirchhoff and Mindlin plate model based on a modified couple stress theory, Compos. Struct. 95 (2013) $142-153$

[25] O. Civalek, B. Akgoz, Free vibration analysis of microtubules as cytoskeleton components: Nonlocal EulerBernoulli beam modeling, Scientica Iranica, Trans. B-Mech. Eng. 17 (2010) 367-375

[26] Sc Pradhan, Buckling analysis and small scale effect of biaxially compressed graphene sheets using non-local elasticity theory, Sadhana 37 (2012) 461-480

[27] B. Akgoz, O. Civalek, Analysis of Micro-Sized Beams for Various Boundary Conditions Based on the Strain Gradient Elasticity Theory, Arch. Appl. Mech. 82 (2012) 423-443

[28] G.R. Kirchhoff, Uber das gleichgewichi und die bewegung einer elastishem scheibe. JFuer die Reine und Angew. Math. 40 (1850) 51-88

[29] T.H. Von Karman, Encyklop die der Mathematischen, edition IV, Wissenschaften, 1910

[30] D.O. Brush, B.O. Almorth, Buckling of bars, plates and shells, McGraw-Hill Book Company, 1980

[31] L.H. Yu, C.Y. Wang, Buckling of rectangular plates on an elastic foundation using the Levy method, AIAA 12 (2008) 3163-3166 